\title{
Modéliser la propagation d'ondes et de vibrations dans les sols
}

\section{J.-F. SEMBLAT}

Université Paris-Est

IFSTTAR

Département Géotechnique,

Risques naturels et Sciences de la Terre

Laboratoire Séismes et Vibrations

Champs-sur-Marne

-U La modélisation de la propagation d'ondes et de vibrations dans les sols peut s'envisager à l'aide d'approches numériques variées (éléments finis, éléments de frontière, éléments spectraux...). Ces méthodes présentent des avantages et des inconvénients différents. Dans les couches de sol de surface, les ondes et les vibrations peuvent être simultanément amplifiées et atténuées du fait du contraste de vitesse entre les couches et de leurs propriétés d'amortissement. Des modèles 2D/3D sont nécessaires dans de nombreuses situations et l'efficacité et la précision des méthodes numériques pour modéliser de fortes hétérogénéités et des géométries complexes peut s'avérer problématique. En outre, les conditions de radiation des ondes à l'infini ne sont pas aisées à contrôler avec les méthodes d'éléments finis ou spectraux alors qu'elles sont explicitement prises en compte avec les méthodes d'éléments de frontière. Différentes méthodes de couches absorbantes (e.g. F-PML, M-PML, CALM) ont été récemment proposées afin de réduire ces réflexions parasites sur les frontières du maillage. Pour les simulations 3D, de récentes avancées de la méthode des éléments de frontière (i.e. formulations multipôle rapide) autorisent des simulations efficaces même pour des domaines étendus. Finalement, les séismes et vibrations forts peuvent induire des effets non linéaires dans les couches de sol. Dans de telles situations, des avancées récentes sur les formulations numériques et les modèles de comportement sont présentées et discutées dans cet article. Elles permettent de réduire le volume de données expérimentales nécessaire à la validation et à l'application de telles approches.

Mots-clés : ondes, séismes, vibrations, dynamique des sols, simulation numérique, propagation, amortissement, éléments finis, éléments de frontière.

\section{Modelling wave propagation and vibrations in soils}

Waves and vibrations in soils can be modelled through various numerical approaches (FEM, BEM, SEM, etc.). All these methods have various advantages and drawbacks. In surficial soil layers, waves and vibrations may be simultaneously amplified and attenuated due to the velocity contrast between these layers or their damping properties. 2D/3D models are needed in many situations and the efficiency/accuracy of the numerical methods to account for strong heterogeneities and complex geometries is in question. Furthermore, the radiation conditions at infinity are not easy to handle with finite/spectral elements whereas it is explicitly accounted in the Boundary Element Method. Various absorbing layer methods (e.g. F-PML, M-PML, CALM) were recently proposed to attenuate spurious wave reflections at the mesh boundaries. For $3 \mathrm{D}$ simulations, recent advances in the Boundary Element Method (namely Fast Multipole formulations) allow efficient large scale computations. Finally, strong earthquakes or vibrations may involve nonlinear effects in surficial soil layers. Recent advances in numerical formulations and constitutive models in such complex situations are presented and discussed in this paper. A crucial issue is the availability of the field/laboratory data to feed and validate all these models. 


\section{Modélisation numérique de la propagation d'ondes et de vibrations}

Les problèmes de propagation d'ondes sont caractérisés par différents phénomènes (Eringen, 1974; Semblat et Pecker, 2009) : dispersion, diffraction, amortissement, conversions de type d'ondes... La plupart de ces caractéristiques sont rarement accessibles directement par l'expérience. Il est généralement nécessaire de recourir à des expérimentations modèles (matériaux modèles, essais à échelle réduite (Chazelas et al., 2003; Semblat et Luong, 1998)) ou d'utiliser le calcul numérique et/ou des méthodes inverses (Bui, 1993 ; Semblat et al., 2000) afin de déterminer les paramètres caractérisant le matériau et les ondes qui s'y propagent.

Plusieurs méthodes numériques permettent de simuler les phénomènes de propagation d'ondes (Fig. 1) : différences finies (Moczo et al., 2002 ; Virieux, 1986), éléments finis (Joly, 1982 ; Semblat, 1998), éléments de frontière (Bonnet, 1999 ; Dangla et al., 2005), éléments spectraux (Faccioli et al., 1997 ; Komatitsch et al., 1999). Suivant les applications visées, ces méthodes numériques présentent des avantages et des inconvénients différents.

La méthode des éléments finis (Fig. 2, gauche) est très puissante car elle permet de modéliser des géométries et des comportements complexes. Pour les problèmes de propagation d'ondes, elle présente toutefois deux inconvénients principaux : la réflexion d'ondes parasites sur les frontières du domaine maillé (Chadwick et al., 1999 ; Collino, 1996 ; Modaressi et Benzenati, 1992) et la dispersion numérique des ondes (Ihlenburg et Babuška, 1995 ; Semblat et Brioist, 2000). La dispersion numérique provoque une variation artificielle de la vitesse de propagation des ondes en fonction des caractéristiques du modèle d'éléments finis.
En effet, le maillage doit par exemple être suffisamment fin pour bien décrire la forme de l'onde (Fig. 2, gauche).

La méthode des éléments de frontière (Fig. 2, droite) présente l'avantage de permettre une modélisation aisée de la propagation d'ondes en milieu infini ou semi-infini (Bonnet, 1999 ; Dangla et al., 2005). Les conditions de radiation des ondes à l'infini sont en effet directement prises en compte dans la formulation. Par ailleurs, la méthode des éléments de frontière résout le problème aux interfaces entre milieux de caractéristiques homogènes : elle permet donc un gain sensible pour la modélisation de la propagation bidimensionnelle (interfaces unidimensionnelles) ou tridimensionnelle (interfaces surfaciques), mais est donc limitée à des milieux faiblement hétérogènes. Des formulations accélérées de la méthode ont été proposées récemment (Chaillat et al., 2009) ; elles sont présentées dans la suite.

Afin de bénéficier des avantages de ces deux méthodes, il peut être intéressant de les combiner en réalisant un couplage éléments finis/éléments de frontière (Dangla, 1989).

\section{2}

\section{Modélisation de la propagation d'ondes par éléments finis ou spectraux}

\subsection{1}

\section{Dispersion numérique}

La méthode des éléments finis et la méthode des éléments spectraux permettent de modéliser la propagation d'ondes et de vibrations dans les sols. Comme pour d'autres méthodes de simulation, deux types d'er- (a)

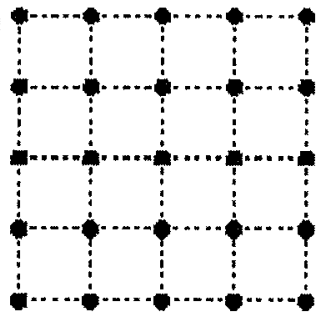

(d)

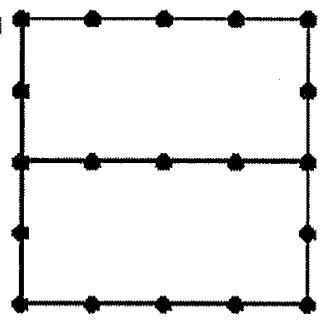

(b)

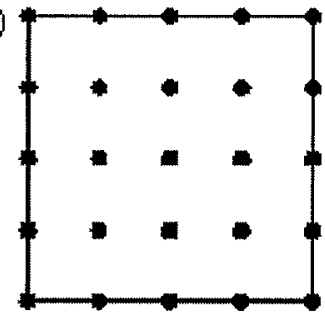

(e)

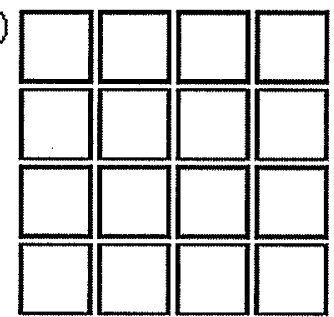

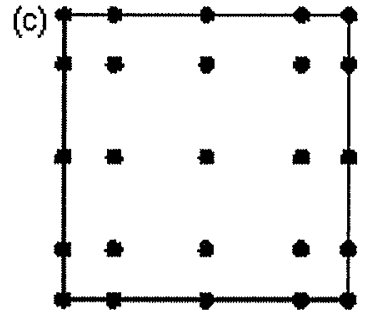

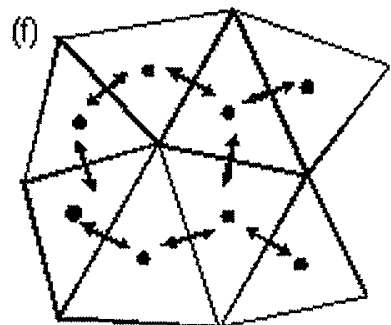

Différentes méthodes numériques permettant de modéliser la propagation d'ondes et de vibrations : (a) méthode des différences finies, (b) méthode des éléments finis, (c) méthode des éléments spectraux, (d) méthode des éléments de frontière, (e) méthode des éléments discrets, (f) méthode des volumes finis. 


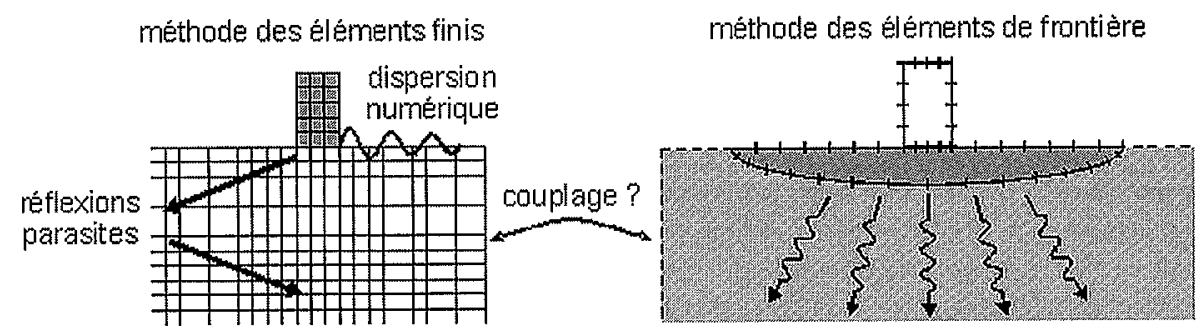

Méthode des éléments finis (gauche) et méthode des éléments de frontière (droite) pour les problèmes de propagation d'ondes et de vibrations.

reur numérique peuvent être étudiés (Hughes, 1987 ; Chaljub et al., 2007 ; Ihlenburg et Babuška, 1995) : l'erreur sur le temps de propagation (erreur en période relative) ; l'erreur sur l'amplitude (liée à l'amortissement numérique). L'erreur sur le temps de propagation varie d'un schéma d'intégration à l'autre (Hughes, 1987). Cette erreur influence donc l'estimation des vitesses de propagation et est appelée dispersion numérique. Cette erreur est appelée dispersion numérique en référence à la dispersion physique qui induit une dépendance entre vitesses de propagation et fréquence (Deraemaeker et al., 1999; Hughes et al., 2008; Ihlenburg et Babuška, 1995 ; Semblat et Brioist, 2000). La qualité d'une simulation de propagation d'ondes dépend donc du schéma numérique au travers des paramètres suivants : taille des éléments, schéma d'intégration, degré d'interpolation des éléments, etc. Il est nécessaire de contrôler la précision du schéma numérique utilisé car l'erreur numérique tend à s'accroître au cours de la propagation.

Plusieurs travaux théoriques proposent des analyses de l'erreur numérique à partir de l'estimation du nombre d'onde approché (Deraemaeker et al., 1999 ; Hughes et al., 2008 ; Semblat et Pecker, 2009). Ainsi, Ihlenburg et Babuška (1995) ont montré qu'il existe une fréquence de coupure au-delà de laquelle le phénomène de propagation ne peut pas être reproduit. Suivant la fréquence d'excitation, l'onde numérique se propage soit plus lentement, soit plus rapidement que l'onde réelle dans le sol ou la solution théorique du problème simplifié. Il est donc nécessaire de contrôler la dispersion numérique des ondes et de quantifier précisément l'erreur numérique.

42

\section{Éléments finis d'ordre élevé et éléments spectraux}

La dispersion numérique des ondes est également influencée par le degré d'interpolation des éléments finis. Les éléments finis d'ordre élevé sont connus pour leur précision dans la simulation des problèmes en élastoplasticité. En acoustique et élastodynamique, plusieurs travaux théoriques proposent des expressions analytiques pour estimer la dispersion numérique (Deraemaeker et al., 1999; Ihlenburg et Babuška, 1995). Des comparaisons entre éléments finis d'ordres faible ou élevé pour simuler la propagation d'ondes ont été récemment proposées par Hughes et al. (2008).

Sur la figure 3 , les résultats de simulations par éléments finis unidimensionnelles (Semblat et

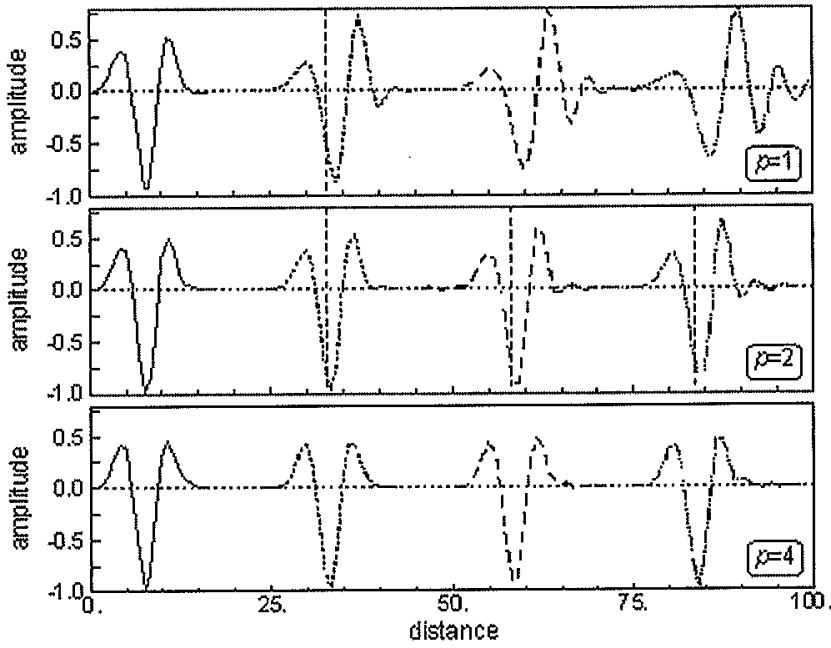

fic. 8 . Dispersion numérique des ondes pour des éléments finis à différents ordres : linéaire (en haut), quadratique (au milieu) et du $4^{\mathrm{e}}$ ordre (en bas) (Semblat, 2000b).

Brioist, 2000) sont présentés pour différents degrés d'interpolation. Le même nombre de degrés de liberté est choisi pour tous les cas. Les éléments finis linéaires (Fig. 3, en haut) conduisent à une forte dispersion numérique (augmentation artificielle de la célérité des ondes). Pour des éléments finis quadratiques (Fig. 3, au milieu), la précision obtenue est satisfaisante par comparaison avec les temps de propagation théoriques (lignes verticales pointillées). Enfin, pour des éléments finis d'ordre élevé (Fig. 3, en bas), la dispersion numérique des ondes est très faible. Ces résultats montrent que, à nombre de degrés de liberté constant, la précision des éléments finis d'ordre élevé pour les simulations la propagation d'ondes et de vibration est bien meilleure que celle des éléments d'ordre faible.

En dehors des éléments finis « classiques », les éléments spectraux sont de plus en plus utilisés car leur précision pour les simulations de propagation d'ondes est très grande (Chaljub et al., 2007 ; Faccioli et al., 1996 ; Komatitsch et Vilotte, 1998). Les éléments spectraux sont généralement choisis à des ordres très élevés (ordres 4 à 8) car le gain de précision par rapport aux éléments finis classiques est faible en deçà de ces ordres. Une évaluation de l'efficacité et de la précision d'éléments spectraux et d'éléments finis à divers ordres ont été proposées par Dauksher et Emery (1999). 


\section{Modélisation de l'amortissement}

L'analyse de la propagation des ondes et vibrations dans un milieu amortissant, comme les sols, nécessite des modèles d'amortissement pertinents et efficaces. L'un des modèles les plus courants en dynamique des structures s'appuie sur la formulation de Rayleigh qui permet de construire la matrice d'amortissement sous la forme d'une combinaison linéaire des matrices de raideur et de masse (Hughes, 1987). L'amortissement obtenu est minimum à une fréquence donnée et tend vers l'infini pour les fréquences faibles et élevées (Chopra, 2007 ; Semblat et Pecker, 2009).

Pour les problèmes de propagation d'ondes, l'amortissement de Rayleigh est équivalent à un modèle rhéologique particulier (modèle de Maxwell généralisé, (Semblat, 1997)). Pour des valeurs d'amortissement faibles ou modérées, cette équivalence permet une estimation simple et explicite des deux coefficients de la formulation de Rayleigh à partir des paramètres de comportement expérimentaux. D'autres formulations (" matricielles ) d'amortissement sont envisageables comme la formulation de Caughey (Chopra, 2007; Semblat et Pecker, 2009) qui correspond à une généralisation de la formulation de Rayleigh et autorise des variations amortissement-fréquence plus complexes.

Différents types de formulations viscoélastiques (mécaniques ou rhéologiques) ont également été proposés pour modéliser l'amortissement. Kjartansson (1979) a étudié des modèles à atténuation (ou facteur de qualité) constante en fréquence (constant $Q$ models) pour les problèmes de propagation d'ondes. Day et Minster (1984) ont considéré une approximation de Padé pour modéliser les milieux amortissants. Emmerich et Korn (1987) ont proposé un modèle rhéologique conduisant à une atténuation quasi constante dans une certaine gamme de fréquence (nearly constant Q). Moczo et Kristek (2005) ont discuté de simulations dans le domaine temporel en utilisant ce type de modèles. Carcione et al. (2002) ont étudié une formulation d'amortissement basée sur la notion de dérivées fractionnaires. Toutes ces approches sont formulées dans le domaine linéaire et nécessitent, pour des simulations en temps, de gérer des variables de mémoire ce qui peut s'avérer coûteux. Des lois de comportement non linéaires peuvent également être nécessaires pour modéliser les vibrations d'amplitude élevée (e.g. vibrofonçage) ou les mouvements sismiques forts (Santini et al., 2008).

\section{Propagation en milieu infini}

Pour les méthodes de différences finies, d'éléments finis et d'éléments spectraux, il est également nécessaire de tenir compte des conditions de rayonnement des ondes à l'infini. Il est en effet indispensable de limiter les réflexions parasites sur les limites du maillage (Bielak et al., 2003). Cela peut être réalisé en mettant en œuvre des conditions absorbantes à la frontière ou des éléments infinis (Chadwick et al., 1999 ; Modaressi et Benzenati, 1994). Cela peut être néanmoins difficile pour les milieux hétérogènes (Chammas et al., 2003). Des méthodes de couches absorbantes ont également été proposées récemment (Basu et Chopra, 2003 ; Festa et Vilotte, 2005). Elles sont discutées en détail ci-après.

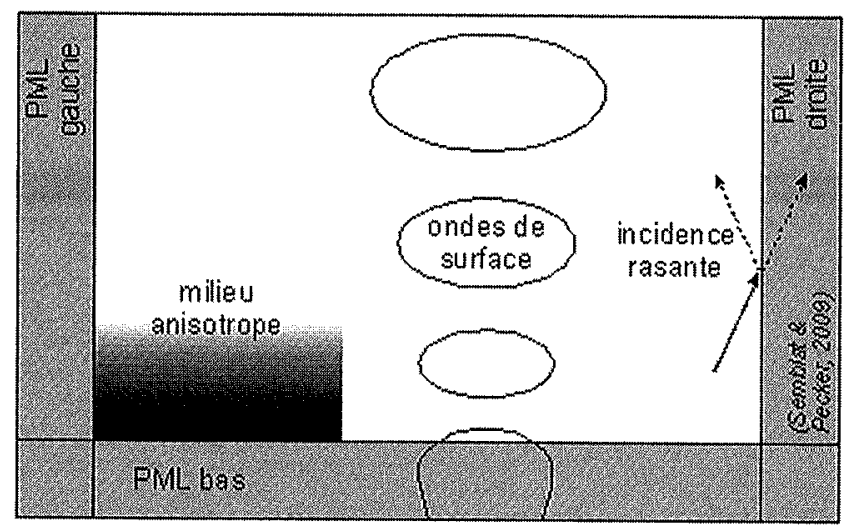

7. l'efficacité et la stabilité des conditions de couches absorbantes ( $d^{\prime}$ après Semblat et Pecker, 2009).

\section{H. \\ Méthodes de couches absorbantes}

Les méthodes de couches absorbantes permettent de limiter les réflexions d'ondes parasites aux frontières du maillage à l'aide d'une couche fictive amortissante (Semblat et Pecker, 2009). Ces méthodes sont de plus en plus utilisées car leur efficacité s'avère très bonne dans de nombreuses configurations. Elles sont généralement connues sous le nom de méthode de couches parfaitement adaptées ou Perfectly Matched Layers (PML).

Différents types de formulations de type PML ont été récemment proposés :

- PML classiques (Basu et Chopra, 2003) : les ondes se propageant dans la couche amortissante sont amorties suivant une loi de décroissance d'amplitude similaire à celle d'un milieu amortissant unidimensionnel, soit :

$$
\tilde{X}=x+\frac{\Sigma(x)}{i \omega}
$$

Cette loi en variable complexe provoque une décroissance d'amplitude de l'onde numérique suivant la direction $x$. Comme illustrée sur la figure 4, pour des incidences rasantes, la réduction d'amplitude sera limitée. Cette loi uniaxiale ne permet donc pas d'obtenir une atténuation suffisante des ondes parasites dans toutes les configurations;

- PML filtrantes (Festa et Nielsen, 2003 ; Festa et Vilotte, 2005) : pour les modèles numériques peu élancés, la formulation PML classique peut également amplifier les ondes de surface (Fig. 4). Une formulation de PML filtrantes (F-PML) a été proposée par Festa et Vilotte (2005) pour remédier à ce problème (Fig. 5) :

$$
\widetilde{x}=x+\frac{\Sigma(x)}{i \omega+\omega_{c}}
$$

- PML multidirectionnelles (Meza-Fajardo et Papageorgiou, 2008) : une autre alternative pour assurer la stabilité numérique des PML (e.g. incidences rasantes) est la formulation de PML multidirectionnelles (M-PML) proposée récemment par Meza-Fajardo et Papageorgiou (2008). 

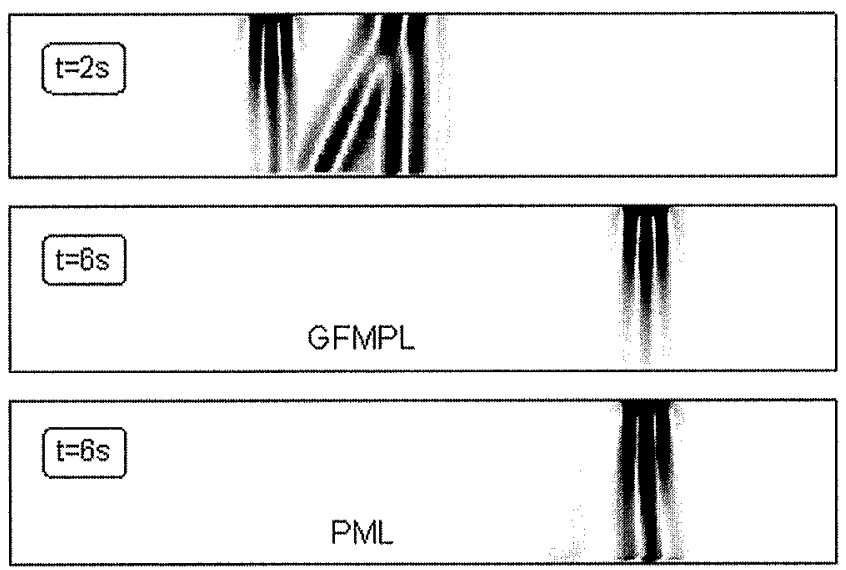

14. Hes 5 Efficacité de la méthode des PML filtrantes proposée par Festa et Nielsen (2003).

La loi de réduction d'amplitude uniaxiale est généralisée au cas multiaxial grâce à l'expression suivante :

$$
\left\{\begin{array}{l}
\widetilde{x}=x+\frac{1}{i \omega} \alpha_{x}^{(x)} X \\
\widetilde{y}=y+\frac{1}{i \omega} \alpha_{y}^{(x)} y \\
\tilde{z}=z+\frac{1}{i \omega} \alpha_{z}^{(x)} z
\end{array}\right.
$$

Il est ainsi possible de choisir un vecteur d'atténuation $\alpha$ permettant d'optimiser l'effet d'atténuation en fonction du type d'onde et de l'incidence. Un autre intérêt de cette formulation est sa stabilité numérique dans le cas de milieux fortement anisotropes (Fig. 4).

Plus récemment, une méthode de couche absorbante simple (appelée Caughey Absorbing Layer Method ou CALM) a été proposée par Semblat et al. (2011). Cette méthode utilise une formulation d'amortissement de type Rayleigh ou Caughey (déjà disponible dans la plupart des programmes éléments finis généralistes) et propose des paramètres d'amortissement optimaux pour différentes configurations (Fig. 6). Comparée aux techniques de type PML, cette méthode s'avère tout à fait efficace (Semblat et al., 2011).

\section{4}

\section{Prise en compte du comportement non linéaire}

Pour modéliser les vibrations ou séismes de forte amplitude, il est nécessaire de mettre en œuvre des lois de comportement non linéaires (Heuze et al., 2004). Pour la propagation d'ondes, il peut être suffisant de reproduire l'une des principales caractéristiques du comportement dynamique des sols : la décroissance du module de cisaillement et l'augmentation de l'amortissement avec le niveau de sollicitation (Seed et al., 1986). Dans le cadre de la méthode des différences finies ou de la méthode des éléments finis, il est possible d'utiliser des modèles s'appuyant sur la loi Masing généralisée (Bonilla, 2000 ; Santini et al., 2008). Líinfluence de l'histoire de chargement et de la pression interstitielle peut également être analysée à l'aide de modèles plus complexes (Aubry et al., 1982 ; Gyebi et Dasgupta, 1992 ; Lade, 1977 ; Loret et al., 1997 ; Mellal et Modaressi, 1998 ; Park et Hashash, 2004 ; Pham et al., 2013; Prevost, 1985).
Au cours des dernières décennies, des modèles (viscoélastiques) linéaires équivalents ont été largement utilisés afin d'avoir une description simplifiée (i.e. peu de paramètres) de la décroissance du module de cisaillement et de l'augmentation de l'amortissement (Schnabel et al., 1972). Des travaux récents proposent de nouveaux modèles simplifiés permettant de limiter les inconvénients des modèles linéaires équivalents tels que l'indépendance vis-à-vis de la fréquence (Assimaki et Kausel, 2000 ; Kausel et Assimaki, 2002). Comme schématisé sur la figure 9 (gauche), ce modèle non linéaire dépendant de la fréquence combine un spectre de déformation avec les courbes $G(\gamma)$ et $\beta(\gamma)$ classiques. Delépine et al. (2007, 2009) ont proposé une autre alternative : un modèle de comportement simple généralisant le modèle à atténuation quasi constante (nearly constant $Q$ (Emmerich et Korn, 1987)) dans le domaine non linéaire. Ce modèle NCQ étendu (ou X-NCQ) conduit à une décroissance du module de cisaillement et à une augmentation de l'amortissement (Fig. 7, droite). Il permet d'analyser la propagation d'ondes sismiques pour des séismes forts et sur des distances importantes (e.g. bassins sédimentaires). In fine, un élément indispensable pour l'ensemble de ces modèles est la disponibilité de données de laboratoire ou d'essais in situ permettant de caractériser le comportement, ou la réponse, non linéaire du sol.

\section{5}

\section{Intérêt de la méthode des éléments de frontière}

\section{5. \\ Principes et limitation de la méthode classique}

La méthode des éléments de frontière (Fig. 2, droite) présente l'avantage de permettre une modélisation aisée de la propagation d'ondes en milieu infini ou semi-infini (Dangla et al., 2005; Chaillat et al., 2009 ; Sheng et Jones, 2006). Les conditions de radiation des ondes à l'infini sont en effet directement incluses dans la formulation. Par ailleurs, la méthode des éléments de frontière résout le problème aux interfaces entre milieux de caractéristiques homogènes : elle permet donc un gain sensible pour la modélisation de la propagation bidimensionnelle (interfaces unidimensionnelles) ou tridimensionnelle (interfaces surfaciques), mais est donc limitée à des milieux faiblement hétérogènes.

Le principal inconvénient de la méthode est qu'elle conduit à des systèmes matriciels pleins et non symétriques. Pour de grands modèles, le coût numérique est alors trop grand. Des travaux récents ont permis de réduire le volume de calcul grâce à une formulation ( multipôle rapide » initialement développée pour les ondes électromagnétiques (Darve, 2000 ; Greengard et al., 1998). Les principes de cette méthode sont présentés ci-après.

\section{9}

\section{Formulation multipôle rapide pour la méthode des éléments de frontière}

Contrairement à la formulation classique qui nécessite de calculer les interactions entre tous les nœuds 


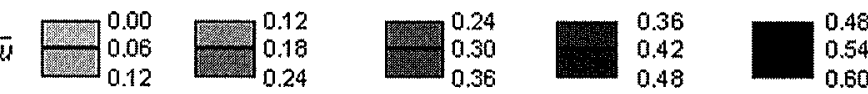
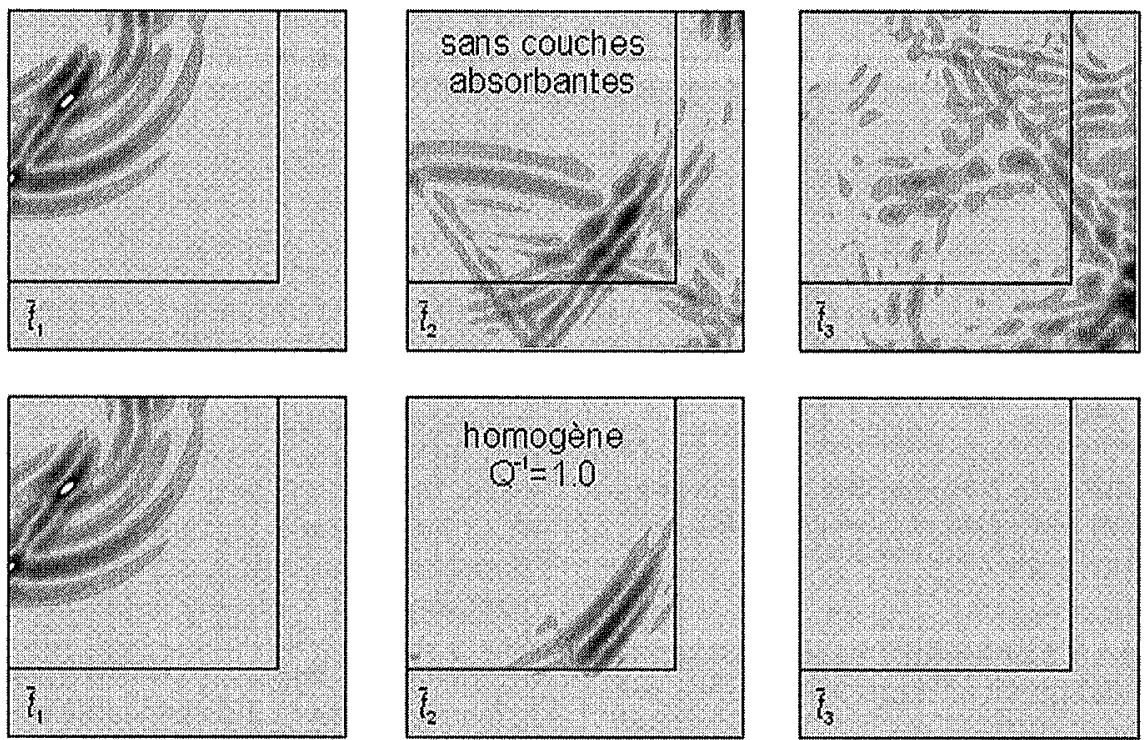

16. 6 Efficacité de la méthode de couches absorbantes CALM : comparaison entre le cas sans couches absorbantes (en haut) et avec couches absorbantes (en bas) (d'après Semblat et al., 2011).
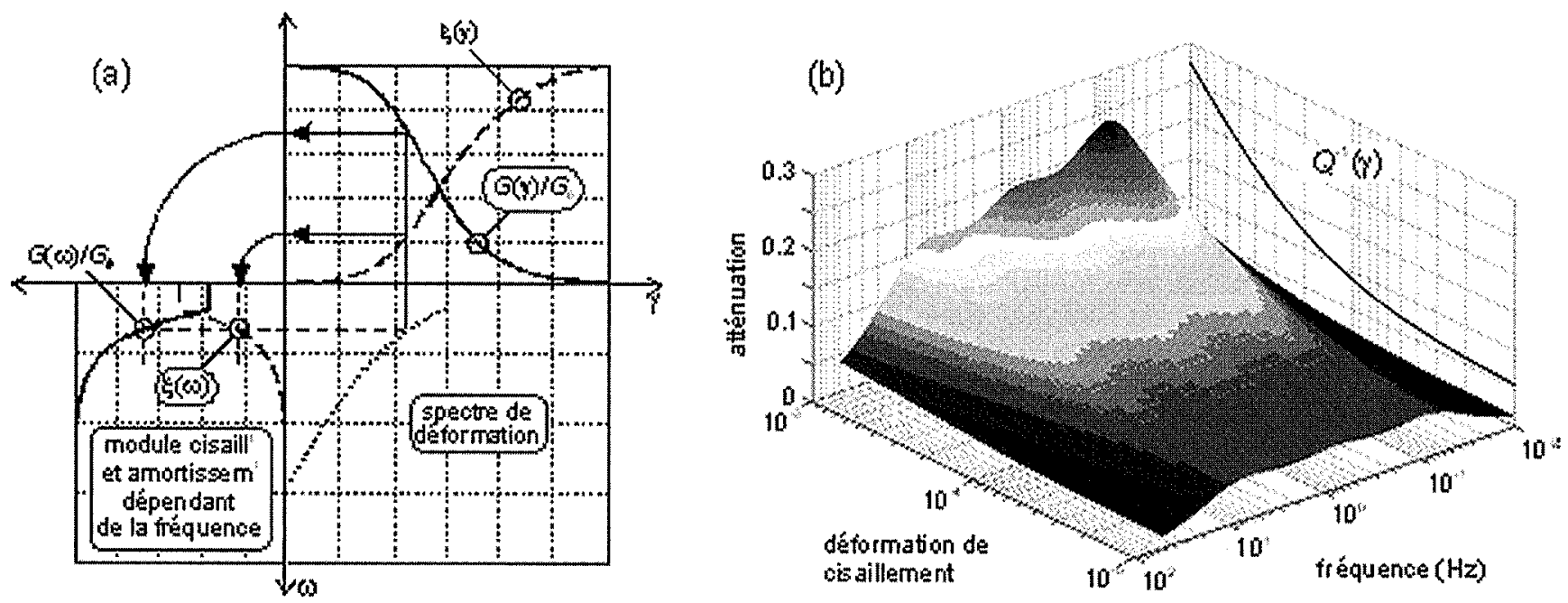

HG. 7 Principes de deux modèles non linéaires simples proposés récemment : (a) modèle dépendant de la fréquence (Kausel et Assimaki, 2002), (b) modèle viscoélastique non linéaire X-NCQ (Delépine et al., 2009).

du maillage par éléments de frontière (Fig. 8, gauche), la formulation multipôle rapide prend en compte les interactions entre groupes de nœuds centrés sur un pôle (Fig. 8, droite). Cela évite de calculer de multiples contributions quasiment identiques car correspondant à des nœuds voisins (Darve, 2000; Greengard et al., 1998 ; Fujiwara, 2000).

Le gain en terme de coût numérique est très important car celui-ci est proportionnel à $\mathrm{N}^{2}$ pour la méthode des éléments de frontière classique (avec $N$ nombre de degré de liberté) et $N \log N$ pour la méthode multipôle rapide (Bonnet et al., 2009 ; Chaillat et al., 2008, 2009). Cette méthode permet donc de simuler des problèmes de grande dimension incluant des millions d'inconnues sur un ordinateur standard. Cela autorise une modélisation plus réaliste des structures géologiques tridimensionnelles et de réaliser des analyses à des fré- quences plus élevées. La méthode a été récemment étendue au cas de la propagation d'ondes en milieu amortissant (Grasso et al., 2012).

\section{5}

\section{Modélisation de l'amplification des ondes sismiques}

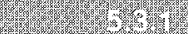 \\ Amplification des ondes sismiques dans les formations sédimentaires}

L'amplification des ondes sismiques dans les couches de sol de sub-surface (ou effets de site) peut 


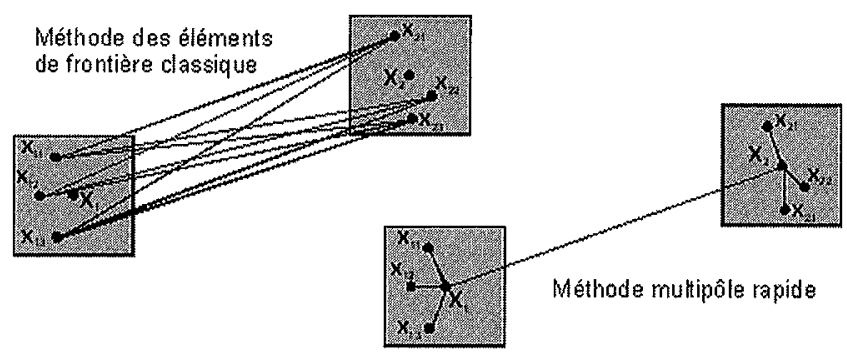

FiG. 8 Comparaison des principes de la méthode des éléments de frontière classique et de la méthode multipôle rapide.

être forte. Le processus d'amplification est contrôlé par le contraste de célérité d'ondes entre les couches, leur géométrie et l'incidence des ondes (Bard et Bouchon, 1985 ; Dobry et al., 1976 ; Duval et al., 1998 ; SanchezSesma et al., 2000 ; Semblat et al., 2000, 2003, 2005). L'amplification des ondes sismiques peut être simulée numériquement à l'aide de la méthode des éléments de frontière. Les principaux phénomènes peuvent ainsi être reproduits :

- amplification due au contraste de célérités entre couches de sol ;

- effets de focalisation des ondes dus à la géométrie complexe des interfaces et de la surface libre ;

- effets de bassin (2D/3D) dus aux ondes piégées dans les couches de surface.

Des effets de diffraction et d'amplification se produisent également autour des irrégularités topographiques marquées telles que les crêtes et les pentes (Bouckovalas et Papadimitriou, 2005 ; Paolucci, 2002 ; Reinoso et al., 1997 ; Sanchez-Sesma, 1983 ; Semblat et Pecker, 2009). Ces phénomènes sont appelés effets de site topographiques et peuvent être simulés par la méthode des éléments de frontière (Semblat et al., 2002).

Les modèles par éléments de frontière permettent d'estimer le facteur d'amplification du mouvement sismique en 2D ou en 3D. Suivant la formulation retenue, l'analyse peut être réalisée soit dans le domaine des fréquences (Bonnet, 1999; Dangla et al., 2005), soit dans le domaine temporel (Gaul et Schanz, 1999 ; Jin et al., 2001 ; Manolis et Beskos, 1988).

\section{4.}

\section{Amplification des ondes sismiques : simulation bidimensionnelle}

Comme de nombreuses mesures ont été réalisées sur le site test européen de Volvi en Grèce (Bard et Riepl-Thomas, 2000 ; Beauval et al., 2003 ; ChavezGarcia et al., 2000 ; Pitilakis et al., 1999), des simulations par la méthode des éléments de frontière ont été entreprises pour ce site (Semblat et al., 2005). Le bassin de Volvi est large de $6 \mathrm{~km}$ et profond de $250 \mathrm{~m}$. Comme le montre la figure 9 , le niveau d'amplification change d'une fréquence d'analyse à l'autre. Le processus d'amplification est influencé par la géométrie du bassin et la stratification du sol (Semblat et al., 2005). Comme la géométrie du bassin est complexe, les ondes se focalisent dans certaines zones ce qui conduit à des niveaux d'amplification élevés (autour de 10) à certaines fréquences (Fig. 9). La géométrie des couches a toutefois une influence forte sur l'amplification à haute fréquence (Fig. 9, en bas). Cette influence a été quantifiée pour un modèle de sol simple ( 2 couches) et un modèle géotechnique détaillé (6 couches) du site de Volvi. Les résultats numériques ont aussi été comparés aux enregistrements de séismes réalisés sur site (Semblat et al., 2005).

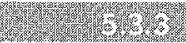 \\ Amplification des ondes sismiques : simulation tridimensionnelle}

Pour simuler l'amplification des ondes sismiques en 3 dimensions, des méthodes numériques efficaces sont nécessaires. Paolucci (2002) a ainsi étudié les effets topographiques sur une colline italienne réelle à l'aide de la méthode des éléments spectraux. La méthode multipôle rapide permet de modéliser des problèmes tridimensionnels. Les effets de site topographiques ont ainsi été analysés pour des configurations tridimensionnelles simples par Chaillat et al. (2008). Comme le montre la figure 10 pour une vallée ellipsoïdale et une onde plane d'incidence oblique, la solution calculée par la méthode multipôle rapide est en très bon accord avec les solutions calculées par Reinoso et al. (1997). Des simulations pour des configurations tridimensionnelles réalistes ont également été effectuées (bassin de Grenoble - Chaillat et al., 2012).
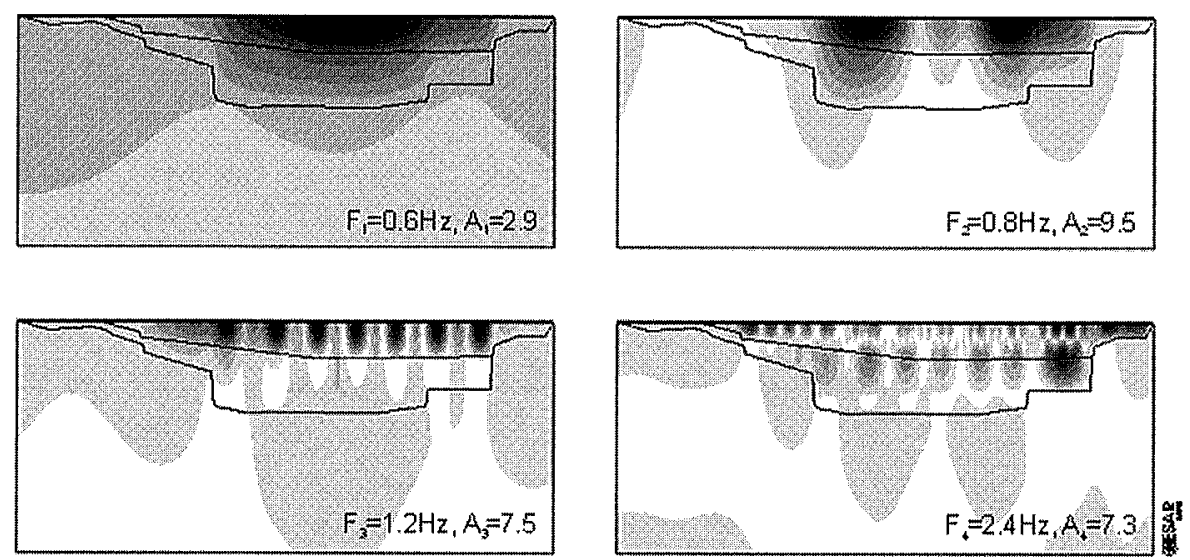

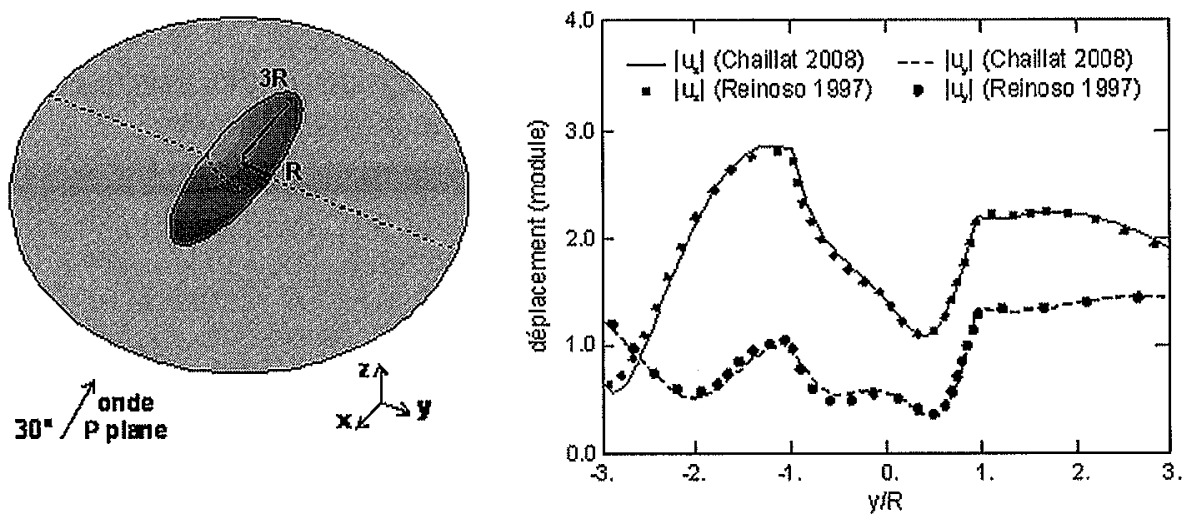

Validation de la méthode multipôle rapide pour une configuration tridimensionnelle simple étudiée par Reinoso (1997) : diffraction d'une onde plane d'incidence oblique par une vallée semiellipsoïdale (Chaillat et al., 2008). Les données correspondant à différentes configurations simples sont disponibles sur http://qsha.unice.fr/

\section{6}

\section{Conclusion}

Cet article présente différentes approches numériques permettant de modéliser la propagation d'ondes et de vibrations dans les sols.

Pour la méthode des éléments finis ou des éléments spectraux, les travaux récents présentés concernent la réduction des réflexions d'ondes parasites aux frontières du modèle. Les méthodes de couches absorbantes (ou PML) ont notamment connu un essor important. Elles permettent de limiter fortement les réflexions d'ondes dans des configurations variées. Un autre point important concerne les ondes et vibrations de forte amplitude. Dans un tel cas, il est nécessaire de recourir à des modèles de comportement non linéaire ce qui est très lourd en dynamique. Des modèles non linéaires simplifiés ont été proposés récemment afin de simuler plus aisément la propagation d'ondes et de vibrations de forte amplitude, et ce sur des distances relativement importantes.
La méthode des éléments de frontière permet de s'affranchir des réflexions d'ondes parasites et de modéliser de façon rigoureuse la propagation d'ondes et de vibrations. La formulation classique de la méthode est toutefois limitée car très coûteuse dans le cas tridimensionnel. Le développement récent de la formulation multipôle rapide permet de dépasser ces limitations et de simuler la propagation d'ondes dans des modèles géotechniques ou géologiques tridimensionnels.

Plusieurs sujets n'ont toutefois pas été discutés dans cet article : influence de la pression interstitielle, interaction dynamique sol-structure ou sol-pieu... (Bielak et Ghattas, 1999 ; Brennan et Madabhushi, 2002 ; Clouteau et Aubry, 2001 ; Groby et al., 2005 ; Semblat et al., 2008 ; Sextos et al., 2003 ; Takahashi et Takemura, 2005). 


\section{Bibliographie}

Aki K., Richards P.G. - Quantitative seismology, Freeman, San Francisco, 1980.

Arulanandan K., Canclini J., Anandarajah A. - Simulation of earthquake motions in the centrifuge. Journal of Geotechnical Engineering, ASCE, vol. 108, n GT5, 1982, p. 730-742.

Bard P.Y., Bouchon M. - The two dimensional resonance of sediment filled valleys. Bulletin of the Seismological Society of America, 75, 1985, p. 519-541.

Bécache E., Fauqueux S., Joly P. - Stability of perfectly matched layers, group velocities and anisotropic waves. Journal Computational Physics, 188 (2), 2003, p. 399-433.

Bielak J., Xu J., Ghattas O. - Earthquake ground motion and structural response in alluvial valleys. Journal of Geotechnical and Geoenvironmental Eng., 125, 1999 , p. 413-423.

Bonnet M. - Boundary integral equation methods for solids and fluids, Wiley, Chichester, UK, 1999.

Bourbié T., Coussy O., Zinszner B. - Acoustique des milieux poreux, Technip-Institut français du pétrole, 1986.

Brennan A.J., Madabhushi S.P.G. - Effectiveness of vertical drains in mitigation of liquefaction. Soil Dynamics and Earthquake Eng., 22 (9-12), 2002, p. 1059-1065.

Bui H.D. - Introduction aux problèmes inverses en mécanique des matériaux, Eyrolles, Paris, 1993.

Cascante G., Vanderkooy J., Chung W. - A new mathematical model for resonantcolumn measurements including eddycurrent effects. Canadian Geotechnical Journal, 42, 2005, p. 121-135.

Chadwick E., Bettess P., Laghrouche O. - Diffraction of short waves modelled using new mapped wave envelope finite and infinite elements. Int. Journal for Numerical Methods in Eng., 45, 1999, p. 335-354.

Chaillat S., Bonnet M., Semblat J.-F. - A multi-level Fast Multipole BEM for 3-D elastodynamics in the frequency domain. Computer Methods in Applied Mechanics and Engineering, 197 (49-50), 2008, p. 4233-4249.

Chaillat S., Bonnet M., Semblat J.-F. - A new fast multi-domain BEM to model seismic wave propagation and amplification in 3D geological structures. Geophysical Journal International, 177 (2), 2009, p. 509-531.

Chaillat S., Semblat J.-F, Bonnet M. - A preconditioned 3-D multi-region fast multipole solver for seismic wave propagation in complex geometries. Communications in Computational Physics, 11 (2), 2012, p. 594-609.

Chazelas J.-L., Guéguen P., Bard P.Y., Semblat J.-F. - Modélisation de l'effet siteville en modèle réduit centrifugé. Validation des techniques expérimentales. $6^{e}$ Colloque national de Génie parasismique, Ecole polytechnique, Palaiseau, 2003, p. 245-252

Chung R.M., Yokel F.Y., Drnevich V.P. - Evaluation of dynamic properties of sands by resonant column testing. Geotechnical Testing Journal, 7 (2), 1984, p. 60-69.

Clouteau D., Aubry D. - Modifications of the ground motion in dense urban areas. Journal of Computational Acoustics, 9, 2001, p. 1659-1675.
Collino F - Conditions aux limites absorbantes d'ordre élevé pour l'équation des ondes 3D. Rapport de Recherche INRIA $n^{\circ} 2932,1996$

Coquel G. - Étude du bruit solidien généré par les transports de surface guidés; impact sur les bâtiments riverains. Thèse de l'École des Ponts, 2008

Dangla P. - A plane strain soil-structure interaction model. Earthquake Engineering and Structural Dynamics, 16, 1988 p. 1115-1128.

Dangla P. - Couplage éléments finis-équations intégrales en élastodynamique et interaction sol-structure. Thèse de doctorat de l'ENPC, 1989.

Dangla P., Semblat J.-F., Xiao H.H., Delépine N. - A simple and efficient regularization method for 3D BEM : application to frequency-domain elastodynamics. Bulletin of the Seismological Society of America, 95 (5), 2005, p. 1916-1927.

Degrande G., Schevenels M., Chatterjee P., Van de Velde W., P. Hölscher, V. Hopman, Wang A., Dadkah N. - Vibrations due to a test train at variable speeds in a deep bored tunnel embedded in London clay. Journal of Sound and Vibration, 293, 2006, p. 626-644.

Delépine N., Bonnet G., Lenti L., Sem blat J.-F. - Nonlinear viscoelastic wave propagation : an extension of Nearly Constant Attenuation models. Journal of Eng. Mechanics (ASCE), 135 (11), 2009, p. 1305-1314

Drnevich V.P., Richart F.E. - Dynamic prestraining of dry sand. Journal of the Soil Mechanics and Foundations Division (ASCE), 96, 1970, p. 453-469.

Emmerich H., Korn M. - Incorporation of attenuation into time-domain computations of seismic wave fields. Geophysics, 9 (59), 1987, p. 1252-1264.

Eringen A.C., Suhubi E.S. - Elastodynamics, Academic press, 1975.

Faccioli E., Maggio F., Paolucci R., Quarteroni A. $-2 \mathrm{D}$ and $3 \mathrm{D}$ elastic wave propagation by a pseudo-spectral domain decomposition method. Journal of Seismology, 1, 1997, p. 237-251.

Festa G., Vilotte J.-P., Delavaud E. - Interaction between surface waves and absorbing boundaries for wave propagation in geological basins : 2D numerical simulations. Geophysical research letters, 32 (20), 2005, p. L20306.1-L20306.4.

Fujiwara H. - The fast multipole method for solving integral equations of threedimensional topography and basin problems. Geophysical Journal International, 140, 2000, p. 198-210.

Gaul L., Schanz M. - A comparative study of three boundary element approaches to calculate the transient response of viscoelastic solids with unbounded domains. Computer Methods in Applied Mechanics and Eng., 179 (1-2), 1999, p. 111-123.

Glinsky-Olivier N., Benjemaa M., Piperno S. Virieux J. - A finite-volume method for the $2 D$ seismic wave propagation. Europ. Geophysical Union General Assembly, Vienna, Austria, April 2-7, 2006

Grasso E., Chaillat S., Bonnet M., Semblat J.-F. - Application of the multi-level time-harmonic fast multipole BEM to 3 -D visco-elastodynamics. Engineering Analysis with Boundary Elements, 36 (5), 2012, p. 744-758.
Greengard L., Huang J., Rokhlin V., Wandzura S. - Accelerating fast multipole methods for the Helmholtz equation at low frequencies. IEEE Comp. Sci. Eng. 5 (3), p. 32-38, 1998

Groby J.P., Tsogka C., Wirgin A. - Simulation of seismic response in a city-like environment. Soil Dynamics and Earthquake Eng., 25 (7-10), 2005, p. 487-504.

Humbert P., Fezans G., Dubouchet A., Remaud D. - CESAR-LCPC, un progiciel de calcul dédié au génie civil. Bulletin des Laboratoires des Ponts et Chaussées, $n^{\circ} 256-257,2005$

Hughes T.J.R. - Linear static and dynamic finite element analysis, Prentice-Hall, Englewood Cliffs, NJ, 1987.

Ihlenburg F., Babuška I. - Dispersion analysis and error estimation of Galerkin finite element methods for the Helmholtz equation. Int. Journal for Numerical Methods in Engineering, 38, 1995, p. $3745-3774$

Joly P. - Les ondes de Rayleigh numériques, Rapport de Recherche INRIA, $n^{\circ} 107$, 1982

Kausel E. - Commentaries on methods to estimate ground motion elicited by fast moving loads. Workshop on Noise and Vibration on High-Speed Railways, Porto, Portugal, 2008.

Komatitsch D., Vilotte J.P., Vai R., CastilloCovarrubias J.M., Sanchez-Sesma F.J. - The spectral element method for elas tic wave equations - Application to 2D and 3D seismic problems. Int. Journal for Numerical Methods in Eng., 45, 1999, p. 1139-1164

Luong M.P. - Mesure des propriétés dynamiques des sols. Revue française de géotechnique, 37, 1986, p. 17-28.

Meza-Fajardo K., Papageorgiou A. - A non-convolutional, split-field, perfectly matched layer for wave propa-gation in isotropic and anisotropic elastic media: stability analysis. Bull. Seism. Soc. Am., 98 (4), 2008, p. 1811-1836.

Moczo P., Kristek J., Vavrycuk V., Archuleta R.J., Halada L. - 3D heterogeneous staggered-grid finite-difference modeling of seismic motion with volume harmonic and arithmetic averaging of elastic moduli and densities. Bulletin of the Seismological Society of America, vol. 92 $n^{\circ} 8,2002$ p. 3042-3066.

Modaressi H., Benzenati I. - An absorbing boundary element for dynamic analysis of two-phase media. 10th World Conf on Earthquake Engineering, Madrid 1992, p. 1157-1163.

Paolucci R., Spinelli D. - Ground Motion Induced by Train Passage. Journal of Engineering Mechanics (ASCE), 132 (2) 2006, p. 201-210

Pham V.A., Lenti L., Bonilla I.F., Semblat J.-F. - Réponse sismique non linéaire des sols et influence de la pression interstitielle. $21^{e}$ Congrès français de mécanique, Bordeaux, 26-30 août 2013.

Santisi d'Avila M.P., Lenti L., Semblat J.-F - Modeling strong seismic motion : 3D loading path vs wavefield polarization. Geophysical Journal International, 190 2012, p. 1607-1624

Saxena S.K., Reddy K.R. - Dynamic moduli and damping ratios for Monterey $n^{\circ} 0$ sand by resonant column tests. Soils and Foundations, 29, 1989, p. 37-51. 
Semblat J.-F. - Rheological interpretation of Rayleigh damping. Journal of Sound and Vibration, 206 (5), 1997, p. 741-744.

Semblat J.-F, Luong M.P. - Wave propagation through soils in centrifuge experiments. Journal of Earthquake Engineering, vol. 2, $\mathrm{n}^{\circ}$ 1, 1998, p. 147-171.

Semblat J.-F. - Amortissement et dispersion des ondes : points de vue physique et numérique. Revue française de génie civil, 2 (1), 1998, p. 91-111.

Semblat J.-F., Brioist J.-J. - Efficiency of higher order finite elements for the analysis of seismic wave propagation. Journal of Sound and Vibration, vol. 231, $\mathrm{n}^{\circ} 2,2000$, p. 460-467.

Semblat J.-F., Duval A.M., Dangla P. Numerical analysis of seismic wave amplification in Nice (France) and comparisons with experiments. Soil Dyna- mics and Earthquake Eng., vol. 19, n ${ }^{\circ} 5$, 2000 , p. 347-362.

Semblat J.-F., Dangla P. - Modélisation de la propagation d'ondes et de l'interaction sol-structure : approches par éléments finis et éléments de frontière. Bulletin des Laboratoires des Ponts et Chaussées, $\mathrm{n}^{\circ}$ 256-257, 2005, p. 163-178.

Semblat J.-F., Kham M., Parara E., Bard P.Y., Pitilakis K., Makra K., Raptakis D. - Seismic wave amplification : basin geometry vs soil layering. Soil Dynamics and Earthquake Eng., 25 (7-10), 2005, p. 529-538.

Semblat J.-H. Yecker A. - Waves and vibrations in soils ; earthquakes, traffic, shocks, constructions works, IUSS Press, 2009.

Semblat J.-F., Lenti L., Gandomzadeh A. - A simple multi-directional absorbing layer method to simulate elastic wave propagation in unbounded domains. Int. Jal Numerical Methods in Eng., 85 (12), 2011, p. 1543-1563.

Sextos A.G., Pitilakis K.D., Kappos A.J. - Inelastic dynamic analysis of RC bridges accounting for spatial variability of ground motion, site effects and soil-structure interaction phenomena. Part 1 : Methodology and analytical tools. Earthquake Engineering and Structural Dynamics, 32 (4), 2003, p. 607627

Takahashi A., Takemura J. - Liquefactioninduced large displacement of pile-supported wharf. Soil Dynamics and Earthquake Eng., 25 (11), 2005, p. 811-825.

Virieux J. - P-SV Wave propagation in heterogeneous media : velocity-stress finite-difference method. Geophysics, vol. 51, 1986, p. 889-901. 